\title{
ACTITUDES DE LOS ESTUDIANTES EN EL APRENDIZAJE DE LA MATEMÁTICA CON TECNOLOGÍA
}

\author{
Gómez-Chacón, Inés Maria \\ Facultad de Ciencias Matemáticas. Universidad Complutense de Madrid \\ igomezchacon@mat.ucm.es
}

Resumen. El creciente uso del ordenador en la enseñanza y aprendizaje de la Matemática en secundaria y bachillerato plantea cuestiones relativas
a las relaciones existentes entre habilidades y actitudes hacia la Matemática y el ordenador. Este artículo examina estas relaciones entre actitudes
hacia la Matemática y hacia la tecnología. Se presenta la respuesta de 392 estudiantes procedentes de cinco institutos diferentes. Los resultados
proporcionan sugerencias para la enseñanza y el aprendizaje de la Matemática en contextos informáticos y la evaluación de actitudes.

Palabras clave. Evaluación de actitudes, actitudes y aprendizaje matemático con tecnología, motivación, programas de geometría dinámica (GeoGebra), educación secundaria.

\begin{abstract}
Students' attitudes to learning mathematics with technology
Summary. The use of computers in the teaching and learning of High School level mathematics raises many as yet unanswered questions about the relationships between students' perceived abilities and attitudes towards mathematics and computers. This paper examines these relationships between attitudes to mathematics and technology. It reports the responses of 392 students from five schools. The outcomes provide suggestions for teaching and learning mathematics and the measure of attitudes.
\end{abstract}

Keywords. Monitoring students' attitudes, Attitude to learning Mathematics with Technology, Teacher Instruction, Motivation, Interactive Geometry Systems (GeoGebra) in the Classroom, Secondary Education.

\section{INTRODUCCIÓN}

El impacto del ordenador es cada vez más creciente a nivel social y escolar. En los últimos estudios sobre la integración de las nuevas tecnologías en el aprendizaje matemático se ha señalado la necesidad de avanzar en aspectos de comprensión y organización de situaciones didácticas (p.e. ver publicaciones ICMI Study $17^{1}$ ). Contamos con importantes resultados de investigaciones cuyo núcleo central ha sido el estudio de propiedades instrumentales y simbólicas de la tecnología en distintas áreas de conocimiento matemático (programas concretos de cálculo simbólico y programas de geometría dinámica) (Artigue, 2002; Gómez-Chacón y Joglar, 2008; Guin y Trouche, 1999; Hohenwarter y Lavicza, 2007; Noss, 2002; Lagrange, 2000; Richard, Fortuny, Hohenwarter y Gagnon, 2007). En muchos de estos estudios se caracteriza la contribución de las TIC al desarrollo de niveles cognitivos (Mariotti, 2002). Sin embargo, son mucho más escasos los estudios que han intentado evaluar los efectos del rol de la tecnología en actitudes y aspectos afectivos.

Una de las razones principales de esta escasez se debe a que este tipo de resultados son difíciles de medir y de comparar. Nosotros sustentamos la tesis de que generar una mejor comprensión y significado matemático tiene una componente afectiva que necesita ser entendida a fin de determinar la forma o las experiencias en las que las tecnologías se pueden utilizar para un mejor aprendizaje matemático. Por tanto, con objeto de dar respuesta a esta necesidad, venimos desarrollando desde 2006 un proyecto en la Facultad de Ciencias Matemáticas de la Universidad Complutense de Madrid. Este proyecto se centra en desarrollar y validar instrumentos para la medida de actitudes hacia el ordenador, especialmente en contextos de Secundaria y con estu- 
diantes universitarios, tratando de identificar el conjunto de actitudes con mayor impacto en los contextos de aprendizaje matemático mediado por software específico como, por ejemplo, el programa GeoGebra (Gómez-Chacón (en prensa) $)^{2}$.

Para este artículo hemos seleccionado una parte de esta investigación, específicamente la parte del estudio que tiene como finalidad dar respuesta a las siguientes cuestiones:

- ¿Qué interacción se produce entre actitudes hacia la Matemática y actitudes hacia la tecnología en estudiantes de secundaria?

- Para el estudio de esta interacción, ¿qué instrumentos de evaluación de actitudes, utilizados en anteriores investigaciones, siguen siendo adecuados (por ejemplo Galbraith y Haines, 1998 y 2000)? ¿Necesitan ser incorporadas otras dimensiones de la actitud?

En síntesis, el propósito del trabajo es estudiar la relación entre actitudes hacia la Matemática y el ordenador en estudiantes de $4^{\circ}$. de secundaria y $1^{\circ}$. de bachillerato (estudiantes de 15-16 años) y mostrar, mediante datos empíricos, algunas dimensiones que pueden contribuir a la evaluación de las actitudes en el aprendizaje matemático en ambientes tecnológicos.

\section{INVESTIGACIONES SOBRE ACTITUDES Y USO DE NUEVAS TECNOLOGÍAS EN EL APRENDIZAJE MATEMÁTICO}

En las últimas décadas se ha desarrollado de forma significativa el estudio de las actitudes en educación matemática, desde las primeras investigaciones centradas en las relaciones entre actitudes positivas y rendimiento (Leder, 1985; Leder y Forgasz, 2006), estudios centrados en la medida de actitudes en distintas áreas de la matemática (Kulm, 1980; Estrada, 2002), metaanálisis y estudios recientes sobre la naturaleza de la actitud (Ruffell et al., 1998), la búsqueda de una buena definición (Di Martino y Zan, 2001, 2002), o la exploración de instrumentos como cuestionarios (Hannula, 2002).

Aunque el estudio de las actitudes hacia la matemática se viene desarrollando desde hace tiempo, el estudio de las actitudes hacia la tecnología en el aprendizaje matemático tiene una historia más corta. Sobre esto, han sido significativas las investigaciones llevadas a cabo con estudiantes universitarios (Galbraith y Haines, 2000; Cretchley y Galbraith, 2002; Camacho y Depool, 2002; Gómez-Chacón y Haines, 2008) y en el ámbito de secundaria (Forgasz, 2003; Goos et al., 2003; Pierce y Stacey, 2004; Pierce, Stacey y Brakatsas, 2007). Se podría afirmar que la tendencia en estos estudios ha sido el uso de los cuestionarios para la evaluación de las actitudes, formulados desde la perspectiva de la definición multidimensional de actitud (cognitiva, afectiva y conductual) y teniendo en cuenta, como principales dimensiones respecto a su influencia en el aprendizaje, la confianza y la motivación matemática. Son más escasos los estudios en los que la actitud investigada haya sido más específica, por ejemplo, valores de la tecnología en el aprendizaje matemático, características del método matemático en contextos tecnológicos, expectativas de éxito y dificultades de uso.

Reseñar que estos estudios han obtenido conclusiones similares, indicando que «existe una débil relación entre actitudes hacia la matemática y actitudes hacia el ordenador (ambas, confianza y motivación) y que las actitudes de los estudiantes en el aprendizaje matemático en contextos tecnológicos correlacionan más fuertemente con las actitudes hacia los ordenadores que con las actitudes hacia las matemáticas» (Cretchley y Galbraith, 2002:8).

\section{MARCO TEÓRICO PARA LA EVALUACIÓN DE ACTITUDES HACIA EL APRENDIZAJE MATEMÁTICO CON TECNOLOGÍA}

En este artículo se ha adoptado la definición multidimensional de la actitud, en la que reconoce tres componentes: respuesta emocional, de creencias y de comportamiento hacia el objeto (Breckler, 1984; Hart, 1989; Hernández y Gómez-Chacón, 1997; Di Martino y Zan, 2001, 2003). Desde este punto de vista, la actitud hacia la matemática se define como una forma articulada por las emociones que el sujeto asocia con las matemáticas (positivas o negativas), por las creencias que tiene sobre las matemáticas y por el comportamiento con el que actúa (Hart, 1989). De acuerdo con esto, una actitud negativa no sólo está caracterizada por una disposición negativa emocional ( «no me gusta la matemática»), sino también por una epistemología incorrecta de la disciplina (es decir, una visión de la disciplina que no es compartida por los expertos).

Al adoptar esta definición multidimensional, en este estudio se puede definir la actitud «negativa» de un estudiante que le gusta la matemática, si esta emoción positiva está asociada con una visión de la matemática como un conjunto de reglas a memorizar.

En la literatura sobre el tema «actitudes hacia el aprendizaje matemático con tecnología» hay consenso entre los expertos (Galbraith y Haines, 2000; Pierce y Stacey, 2004) en que existen una serie de dimensiones clave de evaluación: confianza, motivación y compromiso en matemática, confianza, motivación por el ordenador e interacción entre ordenador y matemáticas. Estas dimensiones son medidas por el Cuestionario Tecnología y Matemáticas que se utiliza en nuestro trabajo de campo (Galbraith y Haines, 2000). De acuerdo con estos autores, describimos brevemente lo que entendemos por estos conceptos.

La confianza en matemáticas (Conf-mat) es una dimensión que el estudiante pone de manifiesto cuando considera que el esfuerzo es un valor, no le preocupa la dificultad intrínseca de la matemáticas, espera lograr buenos resultados y se siente bien con la matemática. Entendemos confianza con el ordenador (Conf-ord) cuando se 
siente seguro en las operaciones efectuadas con éste, cuando cree que puede manejar los procedimientos que requiere su uso, cuando piensa que si comete errores podrá resolverlos por sí mismo.

Se constatará motivación con el ordenador (Mot-ord) cuando el estudiante muestra un alto interés por los ordenadores y encuentra que el aprendizaje con ellos es agradable, cuando reconoce que el ordenador le permite más libertad para la experimentación de nuevas ideas (Galbraith y Haines, 2000). A lo que añadimos que el estudiante mostrará una motivación efectiva cuando se muestren dos categorías de uso del ordenador:

- El valor para el usuario, que incluye el compromiso y el estímulo que conllevan actitudes tales como curiosidad e interés, la credibilidad que añade elementos de valor y relevancia; y el valor de la utilidad (reconocer el valor de la tarea antes de poner los medios para realizarla).

- La expectativa de éxito en su desenvolvimiento técnico y en su satisfacción y efectividad operan como estructuras orientadoras de la acción. Esta expectativa de éxito está vinculada a las formas de comportamiento en el uso del ordenador.

La dimensión de compromiso en matemáticas (Commat) se refiere al comportamiento del estudiante y a la expresión de gestos que manifiestan una implicación responsable en el aprendizaje.

Además, como se ha indicado para el estudio de actitudes hacia el uso de tecnología en el aprendizaje matemático, Galbraith y Haines (1998) definen un constructo que denominan interacción entre ordenador y matemáticas (Inter-mat-ord). Al respecto, afirman que en este contexto «los estudiantes muestran interacción alta entre ordenador y matemáticas cuando piensan que los ordenadores mejoran su aprendizaje, proporcionándoles más ejemplos, ayudándoles en procesos de demostración, les ayudan en el establecimiento de conexiones entre pensamiento algebraico y geométrico» (p. 279).

Ahora bien, pese a reconocer que las anteriores dimensiones son esenciales, en las investigaciones que hemos realizado sobre dimensión emocional en matemáticas (Gómez-Chacón, 2000 a y b) y en las referidas a matemáticas y ordenadores (Gómez-Chacón et al., 2001; Gómez-Chacón y Haines, 2008), en las que diferenciamos tres componentes básicos en las actitudes (cognitivo, afectivo y conductual), se puso de manifiesto que las respuestas afectivas en el aprendizaje con uso del ordenador mediante evaluaciones de cuestionarios son esencialmente cognitivas (de creencia), basadas y determinadas por la experiencia (experiencia no necesariamente vinculada al contexto escolar). Y que sin embargo, cuando las evaluaciones de los sujetos se realizan en situación ( $p$. ej. prácticas con ordenador) las reacciones emocionales están más relacionadas con varios procesos cognitivos y conativos que afectan al desarrollo del pensamiento matemático (procesos creativos e intuitivos, procesos atribucionales, procesos de visualización, procesos de generalización, etc.) y los catalogados como procesos directivos (procesos metacognitivos y metaafectivos) (Goldin, 2000; Gómez-Chacón, 2000a y b).

Así, en esta investigación en el estudio cualitativo nos hemos interesado en dimensiones de afecto local y metaafectivas que se manifestaban en la resolución de problemas con GeoGebra y en las entrevistas. Hemos estudiado estos aspectos en términos del modelo del cuadro 1, tomado de Gómez-Chacón, 2000b: 109-130).

Cuadro 1

Aspectos meta-afectivos en la resolución de procesos matemáticos.

Ruta afectiva 1 (habilita para la resolución) $\rightarrow$ Curiosidad $\rightarrow$ confusión $\rightarrow$ perplejidad $\rightarrow$ estimulo-ánimo $\rightarrow$ placer $\rightarrow$ júbiloalegría $\rightarrow$ satisfacción $\rightarrow$ estructura global (esquemas específicos de representación, estructuras generales de autoconcepto).

Ruta afectiva 2 (limita y detiene el proceso de resolución) $\rightarrow \mathrm{Cu}-$ riosidad $\rightarrow$ confusión $\rightarrow$ perplejidad $\rightarrow$ frustración $\rightarrow$ ansiedad $\rightarrow$ miedo y desesperación $\rightarrow$ estructura global (estructuras generales de autoconcepto, odio y rechazo de la matemática/tecnología).

El significado que tiene la aplicación de este modelo al estudio de casos es que ayuda a una mejor comprensión de cómo actúan procesos, afectos y estrategias cognitivas. Y permite detectar bloqueos y estados emocionales de inestabilidad donde el grado de confusión y percepción de amenaza es significativo, generando altos niveles de ansiedad, y, por tanto, condicionando la dimensión actitudinal.

Por tanto, en relación con el aprendizaje matemático y con el ordenador, además de las dos dimensiones clave de la actitud, la motivación y la confianza, ya señaladas en distintas investigaciones, es importante explorar otras dimensiones que están afectando a la interacción ordenador y matemáticas, de ahí el incluir las creencias sobre matemática, las creencias y actitudes sobre el aprendizaje de la resolución de problema, la aceptabilidad del software concreto en uso y tipología de reacciones emocionales (rutas afectivas).

En resumen, en este estudio, además de la confianza y motivación, se contemplan las siguientes dimensiones o componentes de la actitud:

- Componente afectivo: sentimientos positivos o negativos hacia el uso del ordenador y la resolución de problemas matemáticos, rutas afectivas y estados de ansiedad y bloqueo.

- Componente cognitivo de creencia: se refiere a las expresiones de pensamiento, concepciones y creencias, acerca del objeto actitudinal, en este caso, la matemática $\mathrm{y}$ el ordenador.

- Competencia cognitiva instrumental (técnica): percepción de la propia capacidad sobre conocimientos conceptuales e instrumentales y habilidades intelectuales en las matemáticas y en el uso del ordenador. 
- Valor: utilidad, relevancia y valor percibido del ordenador en la vida personal y académica.

- Comportamiento o manera en la que se dispone el sujeto para usar el ordenador y en particular un software concreto como GeoGebra y estilo cognitivo manifestado.

- Dificultad: se refiere a la percibida del aprendizaje matemático con el ordenador (manejo técnico, etc.). Aunque un estudiante pueda reconocer el valor de una materia, sentir interés hacia la misma (componente afectivo) y pensar que tiene suficientes conocimientos y habilidades (componente cognitivo), también puede considerar la materia como fácil o difícil.

- La expectativa de éxito en su desenvolvimiento técnico y en una satisfacción y efectividad operan como estructuras orientadoras de la acción. Esta expectativa está vinculada a las formas de comportamiento en el uso del ordenador.

Estas dimensiones han vertebrado la selección y elaboración de los distintos instrumentos de actitudes hacia la matemática y el ordenador y para el análisis de datos de los estudios de casos que se describen a continuación.

\section{CONTEXTO Y METODOLOGÍA DE INVES- TIGACION}

El grupo de estudio 392 estudiantes de $4^{\circ}$. de secundaria y $1^{\mathrm{o}}$. de bachillerato procedentes de cinco institutos de secundaria (cfr. cuadro 2). Los centros educativos se eligieron con una representación proporcional de contextos socioculturales y socioeconómicos variados. Todos ellos tienen en común ofrecer en su proyecto curricular la integración de las tecnologías en el aprendizaje matemático y combinar clases tradicionales con clases prácticas en el laboratorio, donde utilizan programas de cálculo simbólico, como Derive, y programas de geometría dinámica, como Cabrí y GeoGebra. Los 13 profesores (5 mujeres y 8 varones) responsables de estos cursos eran personas con formación en estos programas y en nuevas tecnologías. Tres profesores con menos de 5 años de experiencia docente y 10 con más de 15 años de experiencia. Cinco de ellos eran coordinadores de proyectos de aprendizaje matemático en ambientes informáticos en su centro. Como características de los estudiantes, nos interesó reseñar el sexo, el nivel de rendimiento y las elecciones que los estudiantes hacían sobre su orientación futura.

La metodología de investigación utilizada ha sido multimétodo. Las fuentes y procedimientos de recogida de datos fueron variados. De una parte, se propuso un diseño empírico analítico que permitía una exploración y análisis de las relaciones y regularidades de la interacción entre actitudes hacia la matemática y actitudes hacia el uso del ordenador, mediante cuestionarios. Estos cuestionarios habían sido validados y utilizados en otras investigaciones anteriores y cuya fiabilidad de acuerdo al coeficiente de alfa Cronbach eran aceptables (Galbraith y Haines, 2000; Gómez-Chacón et al., 2001) (ver anexos 1 y 2). También se usó metodología cualitativa para el estudio de casos (observaciones de clase, audio-vídeo grabaciones, producciones de los trabajos de los alumnos, entrevistas, etc.) (ver anexos 3 y 4).

Las dimensiones medidas por las escalas que componían los tres cuestionarios aplicados fueron:

- En el cuestionario 1: Tecnología y Matemáticas de Galbraith y Haines (2000): confianza en matemáticas, motivación en matemáticas, compromiso en matemáticas, confianza con el ordenador, motivación para el ordenador, interacción entre matemáticas y ordenador

- En el cuestionario 2: actitudes hacia la resolución de problemas y ansiedad.

Cuadro 2

Número de individuos y porcentajes por instituto, sexo, nivel y rendimiento en matemáticas.

\begin{tabular}{|c|c|c|c|c|}
\hline INSTITUTOS & TOTAL & SEXO & NIVEL & RENDIMIENTO \\
\hline Instituto 1 & $65(17 \%)$ & $\begin{array}{l}\text { Chico } 31(8 \%) \\
\text { Chica } 34(9 \%)\end{array}$ & $\begin{array}{l}4^{\circ} \text {. Secundaria } 17(4 \%) \\
\text { Bachillerato } 48(12 \%)\end{array}$ & $\begin{array}{l}\text { Bajo } 35(9 \%) \\
\text { Medio } 24(6 \%) \\
\text { Alto } 6(1 \%)\end{array}$ \\
\hline Instituto 2 & $100(25 \%)$ & $\begin{array}{l}\text { Chico } 43(11 \%) \\
\text { Chica } 57(14 \%)\end{array}$ & $\begin{array}{l}4^{\circ} \text {. Secundaria } 35(9 \%) \\
\text { Bachillerato } 65(17 \%)\end{array}$ & $\begin{array}{l}\text { Bajo } 22(6 \%) \\
\text { Medio } 64(17 \%) \\
\text { Alto } 14(4 \%)\end{array}$ \\
\hline Instituto 3 & $74(19 \%)$ & $\begin{array}{l}\text { Chico } 34(9 \%) \\
\text { Chica } 7(2 \%)\end{array}$ & $\begin{array}{l}4^{\circ} . \text { Secundaria } 26(7 \%) \\
\text { Bachillerato } 15(3 \%)\end{array}$ & $\begin{array}{l}\text { Bajo } 13(3 \%) \\
\text { Medio } 51(13 \%) \\
\text { Alto } 10(2 \%)\end{array}$ \\
\hline Instituto 4 & $41(10 \%)$ & $\begin{array}{l}\text { Chico } 34(9 \%) \\
\text { Chica } 7(2 \%)\end{array}$ & $\begin{array}{l}4^{\circ} . \text { Secundaria } 26(7 \%) \\
\text { Bachillerato } 15(3 \%)\end{array}$ & $\begin{array}{l}\text { Bajo } 12(3 \%) \\
\text { Medio } 17(4 \%) \\
\text { Alto } 12(3 \%)\end{array}$ \\
\hline Instituto 5 & $112(29 \%)$ & $\begin{array}{l}\text { Chico } 61(16 \%) \\
\text { Chica } 51(13 \%)\end{array}$ & $\begin{array}{l}4^{\circ} . \text { Secundaria } 59(15 \%) \\
\text { Bachillerato } 53(14 \%)\end{array}$ & $\begin{array}{l}\text { Bajo } 22(6 \%) \\
\text { Medio } 63(16 \%) \\
\text { Alto } 27(7 \%)\end{array}$ \\
\hline
\end{tabular}


- En el cuestionario 3: actitudes hacia usos de Internet (Gómez-Chacón et al., 2001).

La puntuación de los ítems fue de cero a cinco (cinco opciones de respuesta: desde «totalmente de acuerdo» hasta «totalmente en desacuerdo»). En este artículo consideraremos de forma explícita los resultados del cuestionario 1 para la descripción del grupo total, sobre actitudes hacia las matemáticas y actitudes hacia la tecnología.

El estudio cualitativo de casos se llevó a cabo con un grupo de 17 alumnos de $4^{\circ}$. curso de secundaria (de entre los 392 estudiantes) componentes de un mismo grupo clase del instituto 1 de secundaria (I1). Se focaliza como caso el grupoclase y seis alumnos de este grupo clase (seis casos), a los que se les hizo un seguimiento más exhaustivo. El grupo fue elegido, además de las facilidades dadas por el centro y de contar con los permisos legales para hacer el estudio, porque sus resultados en el cuestionario 1 mostraban puntuaciones por encima de la media sobre su actitud positiva de interacción entre matemáticas y tecnología. Además, los seis alumnos eran representativos de los cuatro perfiles detectados en las actitudes de interacción entre matemáticas y ordenador (datos aportados por el estudio inicial de los 392 estudiantes).

Estos alumnos, a los que hicimos un seguimiento durante 3 meses, participaban durante ese tiempo en una experiencia de aprendizaje de contenidos de geometría mediante GeoGebra. Además, como ya se ha indicado, con estos estudiantes se tuvieron en cuenta observaciones de clase, audio-vídeo, grabaciones, entrevistas y producción de los trabajos de los alumnos. Mediante estos procedimientos se recabó información sobre aspectos tales como: actitudes hacia la matemática y la tecnología, visión de la matemática, competencia en el uso de la tecnología, interés por la resolución de problemas, estados de ansiedad y bloqueo, procesos cognitivos-emocionales que producen una evaluación al realizar matemáticas con una tecnología concreta (GeoGebra).

En estos últimos años se han desarrollado distintos software de geometría dinámica como son, entre otros, Geometric Supposer, Cabri, The Geometric Sketchpad, Cinderella, GeoGebra etc. En nuestro caso hemos escogido trabajar con GeoGebra, ya que integra de forma dinámica la geometría y la expresión algebraica de objetos gráficos y es un software de libre distribución orientado a la educación de matemáticas en secundaria, aspecto este último que se convierte en una facilidad para los institutos con los que hemos experimentado.

El tratamiento de casos de forma cualitativa nos permite acercarnos a diferentes tipos de evaluación: evaluaciones del estudiante cuando éste no está implicado en la tarea (por ejemplo cuando responde a un cuestionario) y evaluaciones de la situación, tipo de evaluaciones que se producen cuando el estudiante está implicado en la actividad matemática concreta (Cuadro 3). Es importante reseñar que en esta investigación se han tenido en cuenta elementos de triangulación (con fuentes de datos y con instrumentos diversos). Cuando se trata de medir actitudes, no basta con la información que el estudiante da a través de un ítem de un cuestionario, sino que su afirmación necesita ser confirmada mediante la observación del comportamiento cuando resuelve tareas matemáticas, en las entrevistas al estudiante y al profesor.

La tipología de problemas elegidos para trabajar en las sesiones ha sido: problemas de producción de GeoGebradibujos y problemas de demostración y de modelización. Estos problemas exigen conocimiento conceptual y procedimental. Las respuestas requieren un proceso constructivo para llegar a la solución y la búsqueda de alternativas; asimismo, requieren del conocimiento conceptual y de su aplicación, y disponer de información que luego será transferida a categorías gráficas y simbólicas, o bien necesitan procesos de conjeturas, particularización, generalización y modelización (Ver cuadro 3 y anexo 4).

Los resultados de los cuestionarios fueron analizados a través del paquete de análisis estadístico SPSS (versión 11.0) mediante las técnicas de análisis factorial exploratorio y del método de rotación normalización Oblimin con Kaiser. Se calcula la fiabilidad del cuestionario a través del valor alfa de Cronbach y se analiza el comportamiento de cada ítem y de las subescalas con respecto a la fiabilidad, así como el análisis cualitativo. A través del ANOVA se analizan las asociaciones e influencias de las variables sexo, rendimiento, colegio, opción (variables independientes) y los ítems de actitud (variables dependientes). Se completan con análisis de clusters o conglomerados, realizados con el programa SPAD para lo cual se han tomado las variables (subescalas) especificadas en el cuestionario Tecnología y Matemáticas. Las clases se caracterizan por aquellas variables que presentan una media superior o inferior a la media global. Se obtiene un estadístico de comparación de ambas medias, denominado Valor T, que se compara con una distribución normal $\mathrm{N}(0,1)$ para establecer el nivel de significación y cuyo valor absoluto ordena las variables por su importancia en la caracterización de las clases. Si una variable no aparece como característica de una clase, quiere decir que presenta una media similar en la clase y en el total de individuos.

\section{RESULTADOS DEL ESTUDIO}

En este apartado se recogen varios tipos de resultados. En primer lugar, se focaliza en los resultados procedentes de la aplicación del cuestionario Tecnología y Matemáticas de Galbraith y Haines (2000), estudiando los análisis descriptivos de las escalas que lo componen, análisis factoriales y de varianzas. A continuación, se presentan los resultados del análisis de clusters, para el cual se han tomado como variables las escalas especificadas en el cuestionario Tecnología y Matemáticas y se ha enriquecido con la información aportada por los otros cuestionarios aplicados (cuestionarios 2 y 3 ) y por la metodología cualitativa. Finalmente, se describe el estudio de casos que nos aporta una visión más amplia del conjunto de actitudes que tienen mayor impacto en los contextos de aprendizaje matemático con tecnología, y que identifica la interacción que se produce entre actitudes hacia la matemática y actitudes hacia la tecnología en este grupo de estudiantes. 
Cuadro 3

Resumen de un episodio emocional de Rubén en una sesión de clase.

\section{Latas de refrescos (Tipo 2: problemas de modelización)}

Un comerciante vende latas de refrescos. Tiene un almacén donde guarda los paquetes, quiere almacenar el mayor número en el menor espacio posible. ¡Ayúdale!

La figura representa un paquete de 12 latas (sin contar con la altura, en dos dimensiones), las latas tienen un radio de $3,2 \mathrm{~cm}$ aproximadamente.

Problema 1:

1. ¿Cuánto espacio ocupa el paquete? ¿Cuánto espacio ocupan las latas? ¿Qué porcentaje de espacio ocupan las latas en el paquete?

2. ¿Qué ocurre si el radio de la lata es otro distinto? ¿El porcentaje de espacio que ocupan las nuevas latas en su paquete cambia?

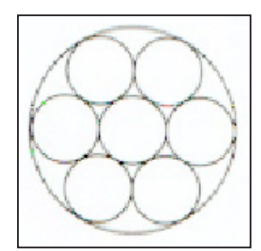

(¿Estás atascado?: intenta pensar con menos latas $(1,2,4,6,8 \ldots)$, o con paquetes más pequeños $1 \times 2,2 \times 3,3 \times 3, \ldots)$

3. Si ahora tienes un paquete de $3 \times 3$, de $2 \times 4$ latas o de $1 \times 1$, averigua el porcentaje de espacio como en la pregunta anterior. ¿Influye el tamaño de la lata en la proporción de espacio que ocupan las latas en el paquete? ¿Y el número de latas?

(¿Estás atascado?: revisa las respuestas que has dado a las preguntas 2 y 3 , puede que te ayuden a encontrar la respuesta correcta).

Comentario: Éste es un problema del tipo 2 de modelización y construcción, forma parte de un proyecto más amplio WebQuest «Latas de Refresco». Tiene como objetivo trabajar la construcción y comprensión de las figuras geométricas, porcentajes y áreas. Se trabajan competencias que favorezcan los procesos de particularización-generalización, demostración, discurso matemático, manejo con GeoGebra de circunferencias, polígonos, tangentes y comprensión algebraica del programa. Trata de poner de manifiesto lo que Kaput distingue entre un medio inerte y uno interactivo por la capacidad del último para responder físicamente a los «input» (Kaput, 1994: 380). Es importante señalar la capacidad de interacción de algunos recursos computacionales, entre ellos el GeoGebra, en el sentido que difieren de los medios inertes porque producen reacciones físicas a las acciones emprendidas sobre ellas; por ejemplo: oprimir la tecla Zoom produce efectos sobre el punto de vista de la gráfica, con el comando Traza; se despliega más información en la ventana de visualización

\section{Episodio emocional de Rubén en la sesión}

Las emociones expresadas por Rubén al inicio de la sesión son de confianza, tranquilidad y diversión. Sin embargo, se bloquea en la resolución, debido al hecho de que no es capaz de pasar a un problema más simple. Trabaja con radio $3,2 \mathrm{~cm}$, en este caso el paquete no entra en el cuadro de visión completo de la pantalla del ordenador, hay que desplazar la ventana para verlo todo. Si hubiera simplificado y tomado como radio 1 , las circunferencias encajan en la cuadrícula perfectamente. Rubén no tomó conciencia de que el comando «zoom» permite alejar o acercar la zona gráfica, pudiendo ver todo el dibujo sin desplazar la ventana.

Rubén insiste en continuar trabajando con radio $3,2 \mathrm{~cm}$. No descubre las ventajas de uso del «zoom» y el tomar de referencia la cuadrícula; por ello, dibuja los círculos y los va colocando a ojo, no situando las circunferencias bien. Muestra dificultad en la identificación del objeto matemático y la representación geométrica. Rubén no es capaz de transferir el proceso de resolución a la pantalla, que él conoce bien tal como lo muestra en lápiz y papel.

La ruta afectiva-emocional expresada por el estudiante: Curiosidad $\rightarrow$ confianza, tranquilidad y diversión. $\rightarrow$ confusión $\rightarrow$ perplejidad $\rightarrow$ frustración $\rightarrow$ ansiedad $\rightarrow$ abandona la tarea con el ordenador $\rightarrow$ estructura global (odio y rechazo de la matemática con tecnología).

En una entrevista posterior, dialogamos con Rubén que claramente había tenido una dificultad instrumental. Como indicamos anteriormente, este problema permite trabajar la doble referencia, una la del manejo del GeoGebra y otra la modelización del problema. Se pone de manifiesto que un deficiente manejo instrumental y las dificultades de transferencia del concepto matemático con la herramienta Geogebra -concepto que maneja bien en la resolución en papel- condicionan la actitud de alumno hacia el ordenador y la interacción entre matemáticas y ordenador.

\subsection{Análisis descriptivos de las escalas del cuestio- nario Tecnología y Matemáticas}

Para el análisis descriptivo hemos considerado las escalas definidas por los autores en el cuestionario Tecnología y Matemáticas (Galbraith y Haines, 2000). El cuadro 4 recoge las medias, desviación típica e índice de fiabilidad de cada escala (Cronbach $\alpha$ ). Los datos ponen de manifiesto que los estudiantes muestran una confianza, motivación y compromiso matemático aceptable ( media $=3,23$, media $=3,08$ y media $=3,3348$ ). Están de acuerdo en que si se esfuerzan obtienen buenos resultados, aunque destacan que las matemáticas les preocupan más que otras asignaturas, reconocen que les pone nerviosos y consideran que las matemáticas son algo abstracto, por tanto, no consideran que sea una asignatura que les divierta tanto, si no le dedican mucho tiempo. 
La confianza, motivación en el uso del ordenador y la interacción del estudiante con la matemática y el ordenador son altas (media $=3,35$, media $=3,28$ y media $=3,30)$. Los estudiantes indican que el ordenador les ayuda a establecer conexiones en matemáticas, por ejemplo una representación gráfica y la ecuación de la misma. Sin embargo, los ítems referidos a formas de trabajo con el ordenador tienen puntuaciones bajas, menores de 3 (no suelen tomar notas de procesos y resultados y no suelen revisar las actividades de matemáticas que han sido realizadas con ordenador).

\subsection{Correlaciones entre motivación, confianza e interacción con el ordenador}

Se consideró cada una de las escalas de actitud como una variable, estudiando las correlaciones entre ellas (Cuadro 5). Se dan correlaciones entre confianza y motivación asociadas con matemáticas $(0,502)$, pero lo están menos con el ordenador $(0,18)$. Se muestra una débil correlación entre confianza matemática y motivación con el ordenador $(0,02)$. El compromiso matemático está fuertemente asociado con la motivación en matemáticas $(0,55)$. La interacción entre ordenadores y matemáticas está asociada con la confianza en el ordenador $(0,56)$; la motivación con los ordenadores $(0,44)$ y con el compromiso en matemáticas $(0,39)$.

Estos datos sugieren que los datos de 5 escalas se articulan en torno a dos ejes (factores) que explican el $69 \%$ de varianza (Cfr. cuadro 6).

En el cuadro 6 se muestran los pesos. La estructura de estos dos factores confirma los resultados de estudios anteriores en los que se afirmaba que ordenador y matemáticas definen diferentes dimensiones con las propiedades dominantes de la escala de interacción. Estos datos parecen ratificar que las actitudes hacia el ordenador y hacia la matemática definen diferentes dimensiones.

Cuadro 4

Medias, desviación típica e índice de fiabilidad de cada escala (Cronbach $\alpha$ ) de las subescalas.

\begin{tabular}{|l|c|c|c|}
\hline \multicolumn{1}{|c|}{ ESCALA } & MEDIA & DESVI. TÍP. & (CRONBACH $\alpha$ ) \\
\hline Confianza en Matemáticas (Confmat) & 3,2341 & 0,65052 & 0,756 \\
Motivación Matemáticas (Motmat) & 3,0800 & 0,64176 & 0,799 \\
Compromiso en Matemáticas (Commat) & 3,3348 & 0,49774 & 0,634 \\
Confianza en los ordenadores (Ordeconf) & 3,3576 & 0,67314 & 0,735 \\
Motivación en los ordenadores (Ordemot) & 3,2861 & 0,47314 & 0,487 \\
Interacción del estudiante con la Matemática y el ordenador (Inter-mat-ord) & 3,3042 & 0,47400 & 0,665 \\
\hline
\end{tabular}

Cuadro 5

Matriz de correlaciones entre las escalas de actitudes.

\begin{tabular}{|l|c|c|c|c|c|c|}
\hline & CONF-MAT & MOT-MAT & COM-MAT & CONF-ORD & MOT-ORD & INTER-MAT-ORD \\
\hline Conf-mat & - & 0,50 & 0,29 & 0,15 & 0,02 & 0,18 \\
Mot-mat & & - & 0,55 & 0,23 & 0,10 & 0,29 \\
Com-mat & & & - & 0,06 & 0,23 & 0,39 \\
Conf-ord & & & & - & 0,82 & 0,56 \\
Mot-ord & & & & & - & 0,44 \\
Inter-mat-ord & & & & & & - \\
\hline
\end{tabular}

Cuadro 6

La matriz de configuración de los factores.

\begin{tabular}{|l|c|c|}
\hline \multicolumn{1}{|c|}{ SUBESCALAS } & 1 & \multicolumn{2}{c|}{ COMPONENTES } \\
\hline Confianza en Matemáticas (Conf-mat) & 0,819 & 0,177 \\
Motivación Matemáticas (Mot-mat) & 0,861 & 0,047 \\
Compromiso en Matemáticas (Com-mat) & 0,636 & 0,340 \\
Confianza en los ordenadores (Conf-ord) & $0,0.5$ & 0,920 \\
Motivación con los ordenadores (Mot-ord) & 0,150 & 0,884 \\
Interacción del estudiante con la Matemática y el ordenador (Inter-mat-ord) & 0,195 & 0,763 \\
\hline
\end{tabular}




\subsection{Análisis de varianza}

A continuación expondremos las actitudes que sostenían los estudiantes, según nivel de rendimiento, centro educativo, curso, opción de futuro y género. Respecto a la variable sexo hubo diferencias significativas en la confianza en matemáticas: las chicas tienen una media más baja $(3,0)$, frente a la de los chicos, aunque son las chicas quienes se comprometen más con el trabajo matemático.

En lo que concierne a rendimiento en matemáticas, se destaca su influencia en la motivación, confianza y compromiso en matemáticas, no siendo significativas respecto a las actitudes hacia el ordenador. Asimismo, los datos por centro educativo (distinto contexto sociocultural) no manifiestan diferencias respecto a la confianza y la interacción con el ordenador, aunque sí respecto a la motivación y el compromiso en matemáticas. Sin embargo, respecto a la interacción entre matemáticas y ordenador y confianza hacia el ordenador se destacan diferencias respecto a los estudiantes que cursan el área de Humanidades, que tienen medias más bajas. Se sienten más confiados los alumnos que desean estudios universitarios de informática que los que desean hacer estudios de $\mathrm{Hu}-$ manidades y de Ciencias.

\subsection{Análisis clusters}

Como indicamos al inicio de este estudio, buscamos una mejor comprensión de cómo se relacionan las actitudes hacia la matemática y las actitudes hacia el ordenador en contextos informáticos de aprendizaje matemático. Para ello, consideramos pertinente un análisis clusters o de conglomerado, utilizando la metodología del programa SPAD. Para el análisis se han tomado como variables las escalas especificadas en el cuestionario Tecnología y Matemáticas y como variables de caracterización el sexo, rendimiento, opción, centro educativo, tipo de dificultades al trabajar la matemática con el ordenador, tipo de sentimiento o emoción que experimenta al resolver problemas matemáticos con el ordenador. Como se ha indicado, además de estas variables que intervienen en la determinación de los clusters, se han estudiado los datos procedentes de los cuestionarios 2 y 3 y que se consideraba que podían completar la información actitudinal de los sujetos que aportaba el cuestionario Tecnología y Matemáticas.

Los resultados ponen de manifiesto que se configuran cuatro perfiles de estudiantes respecto a la interacción entre matemáticas y ordenador:

Perfil 1: Estudiantes con baja interacción entre matemáticas y ordenador, con baja confianza, motivación y compromiso en matemáticas y baja confianza en uso del ordenador. Compuesto por 116 personas (30\%). Son estudiantes con un rendimiento bajo en matemáticas (bajas calificaciones), y dedican escaso tiempo a la utilización del ordenador. En sus respuestas indican que sus mayores dificultades son de uso instrumental, apuntan tener dificultades cuando tienen que transferir la información de las matemáticas de la pantalla del ordenador y les cuesta detallar los pasos que han usado cuando resuelven los problemas de matemáticas con el ordenador. Todo esto les produce una reacción emocional negativa hacia los usos informáticos para trabajar la matemática. Además, si consideramos la información complementaria de los cuestionarios 2 y 3 , estos estudiantes tienen una baja motivación por la resolución de problemas y por Internet, muestran baja confianza en su competencia por las matemáticas y un índice de ansiedad alto en matemáticas.

Perfil 2: Estudiantes con alta motivación, confianza y compromiso en matemáticas, pero, sin embargo, con baja confianza con el ordenador y baja interacción entre matemáticas y ordenador. Formado por 111 personas $(28 \%)$. Son estudiantes con un alto rendimiento en matemáticas (nota alta) pero que prefieren trabajarlas con lápiz y papel. Para ellos, la resolución de problemas les lleva menos tiempo en papel. El ordenador les da más pereza mental, les distrae y les da menos libertad de movimiento a la hora de trabajar la matemática. También, estos estudiantes tienen más alto el índice de ansiedad. En este grupo encontramos una proporción alta en cursos de bachillerato de ciencias. Los datos de los otros cuestionarios nos muestran que este grupo tiene alta competencia en comprensión de resolución de problemas, ven la matemática como resolución de problemas y su motivación por ella es alta. Sin embargo, su índice de ansiedad es más bajo que la media del grupo.

Perfil 3: Estudiantes que tienen alta confianza en uso del ordenador y alta interacción entre la matemática y ordenador, pero con una ejecución más baja en matemática. Constituido por 105 personas (27\%). Son estudiantes a quienes les divierte trabajar con el ordenador y dedican tiempo a su uso. Sin embargo, tienen más baja la confianza, la motivación y compromiso con las matemáticas. En nuestro estudio, las características más significativas de estos estudiantes son su motivación y sus emociones positivas hacia el uso del ordenador (les alegra probar nuevas ideas con él), pero, sin embargo, tienen algunas dificultades de origen cognitivo en el trabajo matemático con el ordenador y les preocupa la asignatura de matemáticas más que otras. Si consideramos los resultados de los cuestionarios 2 y 3, constatamos que este grupo tiene alta motivación por el uso de Internet, su competencia en comprensión matemática es baja, creen que la matemática es memorización y su índice de ansiedad es alto.

Perfil 4: Estudiantes que tienen valores altos en las 5 variables: confianza, motivación, compromiso en matemáticas y confianza y motivación con el ordenador y actitud de interacción ordenador y matemáticas. Compuesto por 60 personas $(15 \%)$. Son estudiantes con una nota alta en matemáticas y con emociones positivas en el uso del ordenador, no manifestando grandes dificultades en su uso. Una proporción alta desea cursar estudios universitarios de informática. Son estudiantes muy motivados por el uso de Internet y por la resolución de problemas matemáticos, dicen tener buena capacidad para la comprensión matemática y muestran escasos elementos de ansiedad. 
En trabajos anteriores se ha estudiado el comportamiento de los estudiantes en el uso de calculadoras gráficas expresando diferentes perfiles de sujetos. Por ejemplo, Goos y otros (2000) categorizan los perfiles de comportamientos según las interacciones en clase y las formas de apropiarse el sujeto de la tecnología en cuatro metáforas según el grado de sofisticación: tecnología como amo o señor, como sirviente, como compañero y como extensión de sí mismo. Mientras que Guin y Trouche (1999) se centran en el método del trabajo del estudiante desde el punto de vista de la génesis instrumental (instrumentalización e instrumentación) y categorizan los sujetos según método de trabajo racional, método inventivo/ingenioso y método teórico. Si comparamos nuestros perfiles con estas categorizaciones, aparecen elementos coincidentes principalmente con el modo de uso y con el método de trabajo. No obstante, nuestros perfiles no son totalmente biyectivos con los perfiles anteriores. En nuestros resultados se pone de manifiesto que hay otros elementos influyentes en la interacción matemática - tecnología como son estados de ansiedad, procesos cognitivos emocionales, motivación por la resolución de problemas y la concepción o creencias que el sujeto tiene sobre qué es la matemática escasamente precisadas en los anteriores estudios (Cuadro 7).

\subsection{Estudio de casos}

En los perfiles anteriores se han identificado, añadiendo a las dimensiones más comúnmente medidas por otros expertos (motivación, confianza, compromiso, interacción matemática y ordenador), otras dimensiones como creencias sobre la matemática y su aprendizaje, actitudes hacia la resolución de problemas y actitudes hacia
Internet y estados emocionales de ansiedad y bloqueo. Ahora bien, pese a que estas últimas dimensiones permitían enriquecer las medidas iniciales del cuestionario Tecnología y Matemáticas, ambas son evaluaciones hechas por los estudiantes sin estar en situación de desempeño de una tarea matemática. Por tanto, surgía la cuestión siguiente: ¿Se mantienen estos perfiles actitudinales cuando el estudiante evalúa en situación (situación de resolución de problemas con un software concreto) y qué diferencias actúan entre los perfiles?

La respuesta es que los datos cualitativos ofrecen variaciones sobre los cambios de actitud y nuevas dimensiones que deben tenerse en cuenta en una evaluación de actitudes hacia la matemática con tecnología, como son por ejemplo las actitudes matemáticas del estudiante y la comprensión de los objetos matemáticos.

Para sustentar esa afirmación se describirán las dimensiones actitudinales de dos de los estudiantes pertenecientes a los casos estudiados, según las categorías apuntadas en el marco teórico y aportadas por el estudio cualitativo. En el cuadro 8 aparecen recogidas las puntuaciones respecto al grupo total y respecto al grupo clase: Rubén (perfil 2) y Alejandro (perfil 4). Según la información procedente del cuestionario 1, ambos son estudiantes con alta motivación, confianza y compromiso en matemáticas, pero que, sin embargo, tienen diferencias tanto en la motivación por el ordenador como en las actitudes de interacción entre matemáticas y ordenador. Estas diferencias hicieron que en el estudio cualitativo nos centráramos en averiguar qué dimensiones hacían que sus actitudes hacia el trabajo matemático con ordenador fueran diferentes.

Cuadro 7

Perfiles actitud matemática y tecnología ansiedad, concepción de la matemática, pensamiento visual.

\begin{tabular}{|c|c|c|c|c|}
\hline \multirow{2}{*}{ Dimensión } & \multicolumn{4}{|c|}{ PERFIL ACTITUD MATEMÁTICA TECNOLOGÍA } \\
\cline { 2 - 5 } & Perfil 1 & Perfil 2 & Perfil 3 & Perfil 4 \\
\cline { 2 - 5 } & $116 \mathrm{e}$ & $111 \mathrm{e}$. & $105 \mathrm{e}$. & $60 \mathrm{e}$. \\
\hline $\begin{array}{c}\text { Bajo nivel de ansiedad } \\
\text { en matemáticas }\end{array}$ & 15 e. (13\%) & 73 e. $(66 \%)$ & 31 e. $(30 \%)$ & 48 e. $(80 \%)$ \\
\hline $\begin{array}{c}\text { Concepción mat- } \\
\text { resoluc-problem }\end{array}$ & 52 e. (45\%) & 83 e. (75\%) & 59 e. $(56 \%)$ & 54 e. $(90 \%)$ \\
\hline Pensamiento visual & Estilo analítico-algebraico & Estilo analítico-algebraico & $\begin{array}{c}\text { Estilo geométrico- } \\
\text { visualizador }\end{array}$ & $\begin{array}{c}\text { Estilo geométrico- } \\
\text { visualizador }\end{array}$ \\
\hline
\end{tabular}

Cuadro 8

Resultados de las puntuaciones de las dimensiones del cuestionario Tecnología y Matemáticas de los casos, del grupo clase y del grupo total.

\begin{tabular}{|l|c|c|c|c|c|}
\hline \multicolumn{1}{|c|}{ ESTUDIANTES } & CONF-MAT & MOT-MAT & COM-MAT & MOT-ORD & INTER-MAT-ORD \\
\hline Rubén & 4,35 & 3,63 & 3,70 & 2,71 & 3,75 \\
\hline Alejandro & 3,63 & 3,50 & 3,88 & 3,86 & 4,63 \\
\hline Resultados grupo-clase (17 alum.) & 3,49 & 3,11 & 3,35 & 3,38 & 3,46 \\
\hline Resultados grupo total de 392 estudiantes & 3,23 & 3,08 & 3,33 & 3,28 & 3,30 \\
\hline
\end{tabular}


A continuación, se presentan de forma comparada los rasgos actitudinales de los dos estudiantes.

- Componente afectivo. Rubén indica que le gusta resolver problemas matemáticos e investigar nuevas ideas. Cuando el estudiante no está implicado en la tarea (se le pregunta mediante cuestionario), considera que es divertido trabajar con el ordenador para su uso personal y para las clases de matemáticas. Sin embargo, cuando está implicado en situaciones y tareas, sus evaluaciones son diferentes, indica que prefiere resolver los problemas sin ordenador y sus sentimientos, cuando utiliza los ordenadores para hacer matemáticas, son de rechazo (un ejemplo es el mostrado en el cuadro 3). Por su parte, Alejandro señala que la matemática es una de las disciplinas que más le agrada, le gusta resolver problemas y perseverar en la búsqueda de las soluciones, y le divierte usar el ordenador. Alejandro, a diferencia de Rubén, cuando está implicado en situaciones concretas resolviendo problemas con GeoGebra, expresa sentimientos de satisfacción y disfrute, y nos comunica que le resulta más cómodo que el aprendizaje de la Matemática por métodos tradicionales. Si comparamos las rutas afectivas-emocionales de ambos sujetos en las prácticas con el ordenador, el número de frecuencias de Rubén de rutas afectivas-emocionales del tipo 2 es mayor que la de Alejandro. En Rubén, desánimo y frustración codifican falta de progreso. Actúa como momento fuerte de confusión y bloqueo, tan paralizante que hace difícil una nueva incorporación a la actividad con el ordenador, por lo que en bastantes casos se pasa a la resolución de problemas con lápiz y papel. Estas rutas configuran en Rubén una estructura de afecto global de rechazo a la realización de matemáticas con el ordenador.

- Componente cognitivo de creencia. Para Rubén las matemáticas son resolver problemas e investigar nuevas ideas, mientras que para Alejandro las matemáticas tienen que ver con resolver problemas, pero también, de forma significativa, con un dominio de conceptos y procedimientos. Ambos estudiantes, en las escalas de actitud, indican que el ordenador les ayuda al estudio de las matemáticas y que utilizar el ordenador hace el aprendizaje matemático más divertido, aunque en el aula Rubén muestra una tendencia clara al uso de lápiz y papel para resolver problemas. Alejandro afirma que GeoGebra le ayuda al estudio de las matemáticas y a establecer conexiones matemáticas.

- Competencia cognitiva instrumental (técnica). Tanto Rubén como Alejandro tienen alta percepción de la propia capacidad sobre conocimientos conceptuales e instrumentales en matemáticas, aunque Rubén muestra baja confianza en su competencia instrumental en el uso del ordenador. A Rubén le falta dominio de ciertos comandos del software, mientras que Alejandro usa las secuencias de comandos y menú del GeoGebra con gran dominio y es capaz de identificar, más que Rubén, el significado del objeto matemático implícito en el comando, estableciendo relaciones entre dibujo y objeto geométrico (matemático). Ambos estudiantes, a veces, tienen dificultades en el uso de algunas representaciones gráficas y en la sintaxis de fórmulas. La capacidad de Alejandro para transferir del papel al ordenador (y viceversa) es alta y en general no tiene dificultad. Además, en las clases con ordenador, este estudiante tiene un alto nivel de comunicación con sus compañeros de trabajo, así como de confianza en sí mismo, y su nivel de investigación en los problemas matemáticos es medio-alto.

- Valor y tiempo de uso. En el caso de Rubén, el tiempo de uso del ordenador en su vida personal y académica es bajo (constatación realizada en la segunda entrevista y en períodos de observación). En la primera entrevista, Rubén había señalado que usaba bastante el ordenador. En el caso de Alejandro, el tiempo de uso del ordenador en su vida personal y académica es alto, aunque en ambos estudiantes el uso del ordenador como herramienta de estudio se limita a la redacción de trabajos y búsqueda de información, sin que hagan un uso específico para las matemáticas.

- Comportamiento o manera en la que se dispone para usar el ordenador y en particular un software concreto como el GeoGebra. Rubén tiene un modo de trabajo estratégico cuando utiliza el lápiz y papel, pero no lo aplica al ordenador en el que actúa con ensayo y error. Su estilo de trabajo se puede estimar un $20 \%$ de componente geométrica y $80 \%$ componente algebraica. Alejandro tiene un modo de trabajo más pautado, sobre todo siguiendo directrices marcadas por el profesor o por él mismo. El estilo de trabajo de este estudiante se puede estimar un $70 \%$ de componente geométrica y visual y $30 \%$ componente algebraica.

- Dificultad. Ambos estudiantes tienen perspectivas diferentes sobre sus dificultades. Rubén considera que el aprendizaje de la matemática con el ordenador es difícil, aunque esto no lo expresa en las escalas de actitudes, sino es algo que se puede constatar en las observaciones de clase. Alejandro considera que el aprendizaje de la matemática con el ordenador es fácil.

- Expectativa de éxito en el trabajo con el ordenador. Rubén tiene una expectativa baja de éxito y piensa que las dificultades técnicas y de transferencias que tiene al usar el ordenador no las puede superar. Por el contrario, Alejandro tiene una expectativa alta de éxito y piensa que superará las dificultades en su desenvolvimiento técnico.

- Evolución después de tres meses. En el caso de Rubén, aumenta su nivel de motivación hacia los ordenadores y por la resolución, al igual que su rendimiento, pero disminuye la confianza y aumenta su ansiedad en matemáticas en contextos tecnológicos. Sin embargo, en el caso de Alejandro los datos muestran una disminución en las puntuaciones de casi todas las subescalas salvo un aumento en su nivel de rendimiento. No obstante, se produce un aumento en su motivación por la resolución y el compromiso en matemáticas, y una disminución en el nivel de ansiedad, lo cual es interpretado como un cambio positivo.

En síntesis, el estudio de casos permite corroborar que, además de las dimensiones de confianza y motivación 
(hacia la matemática y el ordenador), hay otras dimensiones que están actuando fuertemente en la determinación de actitudes y lo que venimos denominando interacción entre actitud hacia la matemática y el ordenador: las creencias que tienen sobre el trabajo matemático, la interacción entre procesos cognitivos-afectivos, la competencia cognitiva instrumental (técnica) y el comportamiento en el uso del ordenador, el valor y expectativa de éxito en el desenvolvimiento técnico y en la satisfacción para una acción efectiva.

En todos los casos estudiados hay un salto en la componente cognitiva de creencia sobre aprender con el ordenador (para todos es divertido) y lo que es convertir el ordenador (artefacto) en un instrumento matemático. Al tratar de profundizar en esta dimensión de «diversión» (como predisposición positiva), encontramos, por ejemplo, en los datos de las entrevistas que el ordenador a nivel personal se usa para chatear y buscar información en Internet, acciones que se sitúan en su espacio de ocio y por eso su evaluación global es positiva. Esto es interesante para comprender por qué muestran en principio una actitud positiva hacia el ordenador. Hay unos elementos de creencia (cognitivos) que están actuando y subyacen en unas experiencias vitales de gestión y evaluación por parte del profesor diferente de las clases (clase tradicional frente a clase en el laboratorio).

Las principales diferencias detectadas entre los casos pertenecientes al perfil 2 y 4 muestran que esta variabilidad en las actitudes están vinculadas al valor y expectativas de éxito, al manejo de estados de ansiedad, a la interacción cognición y afecto, a la percepción que tienen de su competencia instrumental y su forma de comportamiento técnico y de uso; y al estilo matemático del estudiante (geométrico, visualizador, algebraico) que tiene gran influencia en las actitudes matemáticas en contexto informático.

\section{CONCLUSIONES}

La finalidad de este artículo es estudiar las relaciones entre actitudes hacia las matemáticas y el ordenador en estudiantes de $4^{\circ}$. de secundaria y $1^{\circ}$. de bachillerato y mostrar, mediante datos empíricos procedentes del contexto español, algunas dimensiones que pueden contribuir a una más efectiva evaluación de las actitudes en el aprendizaje matemático en ambientes con ordenador. En una primera aproximación a los resultados, se confirman conclusiones que habían sido obtenidas en anteriores investigaciones (Galbraith, 2006), en las que se utilizó el cuestionario Tecnología y Matemáticas, las cuales concluían que era baja la relación entre actitudes hacia la matemática y actitudes hacia el ordenador. Sobre todo, los datos enfatizan que en el aprendizaje matemático usando ordenador hay más fuerte correlación con las actitudes hacia el ordenador que con las actitudes hacia las matemáticas, en particular si se mide la confianza y la motivación hacia las matemáticas y los ordenadores.
Sin embargo, si se tienen en cuenta otras dimensiones diferentes a las medidas por el cuestionario Tecnología y Matemáticas que pueden definir la dimensión actitud de interacción matemáticas con tecnología, tal como quedó expresado en el marco teórico, nuestros datos indican que de las tres dimensiones de la actitud, cognitiva, afectiva y conductual, este instrumento mide más la respuesta emocional en el proceso de aprendizaje matemático con ordenador en su base cognitiva de creencia (evaluaciones y reacciones que tienen que ver con asociaciones del estudiante con el objeto sin estar implicado en la tarea y espacios extraescolares) y menos en la base cognitiva específica de la epistemología matemática en acción. Por tanto, si consideramos la interacción entre actitudes hacia la matemática y hacia el ordenador, junto a las dimensiones de motivación, confianza y el constructo de interacción tecnología y matemáticas definido por Galbraith y Haines (1998), es importante tener en cuenta otras variables.

El análisis global de los resultados a través de los clusters mostró diferentes perfiles de estudiantes. Estos perfiles indican una relación entre estilos de trabajo con el ordenador y actitudes matemáticas de los estudiantes en aprendizaje matemático con tecnología. La tipología de actitudes observada tiene un carácter marcadamente cognitivo y se refiere al modo de utilizar capacidades y procesos que son importantes en el trabajo en matemáticas en contexto informático, es decir, actitudes matemáticas, no actitudes hacia la matemática, lo que apunta como resultado a la relación entre el dominio técnico, procesos de representación-visualización y cuestiones emocionales como un elemento clave para la actitud. Algunos de estos aspectos -los de dominio técnico- también fueron señalados en el estudio realizado por Pierce y Stacey (2004), al indicar que deberían ser incorporados si se quieren evaluar las actitudes de los estudiantes de secundaria.

Por tanto, parece necesario complementar las dimensiones de estas escalas con otras categorías de uso del ordenador que influyen en la actitud del estudiante, en particular con: actitudes matemáticas (carácter cognitivo), con el valor para el usuario, la expectativa de éxito en su desenvolvimiento técnico y en su satisfacción y efectividad, operan como estructuras orientadoras de la acción, y se complementan también con dimensiones de actitudes hacia las matemáticas, como son las relativas a la resolución de problemas y a los estados de ansiedad y bloqueo en situaciones reales de prácticas.

En el estudio de casos llevado a cabo con aprendizaje matemático con GeoGebra, se han constatado diferentes procesos cognitivos-emocionales que producen una evaluación positiva o negativa por parte de los alumnos al realizar matemáticas con tecnología. Se podría decir que estos episodios permiten evidenciar cómo las evaluaciones que sustentan la actitud están estrechamente relacionadas con la competencia cognitiva instrumental o técnica y con el manejo conceptual en matemáticas, aspecto no medido por el cuestionario Tecnología y $\mathrm{Ma}$ temática, lo que hace que se obtenga como resultado una baja correlación entre actitudes hacia la matemática y actitudes hacia la tecnología. 
Además, es importante tener en cuenta las discrepancias según las fuentes de datos respecto a la componente cognitiva de creencia. El sujeto, cuando no está implicado en una tarea concreta y un cuestionario es el estímulo para indagar su actitud, tiene reacciones emocionales que tienen que ver con asociaciones y experiencias previas con las clases de matemáticas y con los ordenadores (en este grupo, principalmente experiencias positivas de dominio de Internet). Asimismo, la componente cognitiva de creencia cambia cuando el estudiante está inmerso en la resolución de una tarea concreta. En esta situación la confianza en sus capacidades está en estrecha dependencia con el dominio técnico del software para los objetos matemáticos. Cuando tiene que enfrentarse a tareas concretas, el alumno percibe el aprendizaje con otra exigencia. Los procesos de instrumentalización dirigidos hacia el artefacto (físico o simbólico) son claves y conducen a la formación de los esquemas de uso y sus actitudes hacia el uso. Quienes más satisfacción y disfrute expresaban por su trabajo con GeoGebra eran estudiantes con mucho más desarrollo de su componente visual (o con un estilo de aprendizaje visualizador en la resolución de problemas) y con un dominio matemático y técnico para el tratamiento de los objetos y las construcciones dinámicas. Quienes expresaban emociones de insatisfacción e indiferencia era debido a que no tenían éxito en su resolución o que trasladaban dibujos tal como quedan reflejados en lápiz y papel (forma estática e inerte), mostrando una falta de diferenciación entre las relaciones entre el dibujo y el objeto geométrico. Esto supone una llamada de atención a que estas actitudes matemáticas no dependen sólo de una actitud inicial positiva o decisiones tomadas espontáneamente por los alumnos, sino que necesitan un aprendizaje explícito y una articulación de esquemas instrumentales en el aula por el profesor.

Estos resultados complementan el trabajo anterior de Galbraith y Haines (2000) e invitan a la elaboración de nuevos instrumentos que tengan en cuenta estos perfiles y se centren en el uso de software concreto.

En síntesis, teniendo en cuenta el marco de referencia que emerge de los datos, se identifican distintos constructos que deberían estar integrados en una evaluación de las actitudes hacia la matemática con tecnología: afectos (emociones, sentimientos hacia el ordenador); cogniciones (evaluaciones, percepciones e información respecto al ordenador); voluntad de acción (intenciones de comportamiento y acciones con respecto al ordenado; comportamiento percibido (soltura o dificultad en el uso del ordenador) y percepción de la utilidad respecto a sus metas globales (el grado en el que el individuo considera que será útil en el futuro). A todo esto es importante añadir que en el estudio de la dimensión actitudinal no basta sólo con el estímulo de actitudes iniciales de interés, de apreciación y gusto, sino que son necesarias las actitudes matemáticas que denotan formas de proceder matemático con herramientas informáticas.

Y para terminar, en orden a evitar inapropiadas generalizaciones, este estudio deja abiertas algunas cuestiones a tener en cuenta en los análisis cualitativos, como son la influencia del tipo de actividades planteadas y la influencia de la gestión del profesor. Ya ha sido informado ampliamente en la literatura sobre tecnología y matemáticas que el diseño de tareas puede ser determinante para el éxito o fracaso del aprendizaje con programas de geometría dinámica y de calculadoras gráficas. En nuestro caso, la elección de actividades puede determinar factores de confianza en el uso técnico del sujeto y en su desarrollo de actitudes.

\section{AGRADECIMIENTOS}

Queremos expresar nuestra gratitud a los estudiantes y profesores involucrados en el estudio y en particular a Cristina Pérez, que tan eficazmente colaboró en la recogida de datos. También, agradecemos el soporte económico recibido en el 2007 del Programa Erasmus Teaching Staff para la estancia de investigación y docencia en la Université de Paris 7 y por el proyecto ASTROCAM (referencia S-0505/ESP/000237) subvencionado por la Dirección General de Universidades e Investigación de la Consejería de Educación de la Comunidad de Madrid para la estancia de investigación en City University London en Londres; ambas estancias nos permitieron profundizar en el marco teórico de esta investigación y discutir con otros expertos.

\section{NOTAS}

1. El texto de discusión asociado a este estudio es accesible en el sitio de ICMI : www.mathunion.org/ICMI/ Digital Technologies and Mathematics Teaching and Learning: Rethinking the Terrain. Study Volumen publicado por Springer en el 2009.

2. Hacemos notar que en este artículo se usa el término tecnología para denotar principalmente herramientas de análisis y uso de programas de ordenador de cálculo simbólico (p.e.. Derive, MathLab) y programas de Geometría dinámica (p.e. Cabri, GeoGebra) para el aprendizaje matemático. Se ha querido mantener este término para respetar la acuñación estándar realizada en los instrumentos de evaluación de procedencia internacional utilizados en el trabajo de campo. 


\section{REFERENCIAS BIBLIOGRÁFICAS}

ARTIGUE, M. (2002). Learning mathematics in a CAS environment: The genesis of a reflection about instrumentation and the dialectics between technical and conceptual work. International Journal of Computers for Mathematical Learning, 7, pp. 245-274.

BRECKLER, S.J. (1984). Empirical validation of affect, behavior, and cognition as distinct components of attitude. Journal of Personality and Social Psychology, 47(6), pp. 1191-1205.

CAMACHO, M. y DEPOOL, R. (2002). Students' attitudes towards mathematics and computer when using DERIVE in the learning of Calculus concepts. The International Journal of Computer Algebra in Mathematics Education, 9(4), pp. 259-283.

CRETCHLEY, P. y GALBRAITH, P. (2002). Mathematics or computers? Confidence or motivation? How do these relate to achievement?, Proceedings 2nd International Conference on the Teaching of Mathematics (Undergrad.), CD and online, Wiley, Crete.

DI MARTINO, P. y ZAN, R. (2001). Attitude toward mathematics: some theoretical issues, en van den Heuvel-Panhuizen, M. (ed.). Proceedings of $25^{\text {th }}$ Conference of the International Group for the Psychology of Mathematics Education, 3, pp. 351-358. Utrecht, Netherlands.

DI MARTINO, P. y ZAN, R.(2002). An attempt to describe a 'negative' attitude toward mathematics, en P. Di Martino (ed.). MAVI European Workshop MAVI XI, Research on Mathematical Beliefs, Proceedings of the MAVI-XI European Workshop, pp. 22-29. University of Pisa, Italy.

ESTRADA, A. (2002). Actitudes hacia la Estadística e instrumentos de evaluación, en: Actas de les Jornades Europees d'Estadística, pp. 369-384. Instituto Balear de Estadística. Palma de Mallorca.

FORGASZ, H.J. (2003). Girls, boys, and computers, for mathematics learning. Girls, boys, and computers for mathematics learning. Keynote address, Annual conference of the Mathematical Association of Victoria <http://www.mav.vic. edu.au/pd/confs/2003/papers/Forgasz_paper.pdf>.

GALBRAITH, P. y HAINES, C. (1998). Disentangling the nexus: Attitudes to mathematics and technology in a computer learning environment. Educational Studies in Mathematics, 36, pp. 275-290.

GALBRAITH, P. y HAINES, C. (2000). Mathematics-computing Attitudes Scales. Monographs in Continuing Education. City University London.

GALBRAITH, P. (2006). Students, mathematics, and technology: assessing the present -challenging the future. International Journal of Mathematical Education in Science and Technology, 37(3), pp. 277-290.

GOLDIN, G.A. (2000). Affective pathways and representation in mathematical problem solving. Mathematical thinking and learning, 2(3), pp. 209-219.

GÓMEZ-CHACÓN, I.Ma . (2000a). Affective influences in the knowledge of mathematics. Educational Studies in Mathematics, 43(2), pp. 149-168.
GÓMEZ-CHACÓN, I.Ma . (2000b). Matemática emocional. Madrid: Narcea.

GÓMEZ-CHACÓN, I.Ma., FIGUEIRAS L. y MARÍN M. (2001). Matemáticas en la red. Internet en el aula de Secundaria. Madrid: Ministerio de Educación, Cultura y Deporte y Editorial Narcea.

GÓMEZ-CHACÓN, I.Ma . y HAINES, C. (2008). Students' attitudes to mathematics and technology. Comparative study between the United Kingdom and Spain. En ICME-11, 11th International Congress on Mathematical Education <http:// tsg.icme11.org/tsg/show/31>.

GÓMEZ-CHACÓN, I.Ma . y JOGLAR, N. (2008). Escenarios multimedia para aprender a enseñar matemáticas con nuevas tecnologías. Estudio de casos. Actas V Congreso Iberoamericano de Docencia Universitaria. Universidad Politécnica de Valencia. CD-Rom.

GÓMEZ-CHACÓN, I.Mª (en prensa). Mathematics attitudes in computerized environments. A proposal using GeoGebra, en Bu, L., Schoen, R., Hohenwarter, M. y Lavicza, Z. Model-centered Learning with GeoGebra: Theory and Practice in Mathematics Education. Sense Publisher.

GOOS, M., GALBRAITH, P., RENSHAW, P. y GEIGER, V. (2000). Reshaping teacher and student roles in technologyenriched classroom. Mathematics Educational Research Journal, 12(3), pp. 303-320.

GOOS, M., GALBRAITH, P., RENSHAW, P. y GEIGER, V. (2003) Perspectives on technology mediated learning in secondary school mathematics classrooms. Journal of Mathematical behavior, 22, pp. 73-89.

GUIN, D. y TROUCHE, L. (1999). The complex process of converting tools into mathematical instruments: the case of calculators. International Journal of Computers for Mathematical Learning, 3(3), pp. 195-227.

HANNULA, M. (2002). Attitude toward mathematics: emotions, expectations and values. Educational Studies in Mathematics, 49, pp. 25-46.

HART, L. (1989). Describing the Affective Domain: Saying What We Mean, en McLeod, D.B. y Adams, V.M. (eds.). Affect and Mathematical Problem Solving, pp. 37-45. Springer Verlag.

HERNÁNDEZ, R. y GÓMEZ-CHACÓN, I.Mª (1997). Las actitudes en educación matemática. Estrategias para el cambio. Revista de Didáctica de las matemáticas, UNO, Monográfico Actitudes y Matemáticas, 13, pp. 41-61.

HOHENWARTER, M. y LAVICZA, Z. (2007). Mathematics teacher development with ICT: towards an International GeoGebra Institute, en Küchemann, D. (ed.). Proceedings of the British Society for Research into Learning Mathematics, 27(3), pp. 49-54. University of Northampton, UK: BSRLM.

KULM, G. (1980). Research on Mathematics Attitude, en Shumway, R.J. (ed.). Research in mathematics education, pp. 356-387. Reston, VA: NCTM.

KAPUT, J. (1994). The representational Roles of Technology in Connecting Mathematics with Authentic Experienc, 
en Biehler, R. Scholz, R.W., Sträßer, R. y Winkelmann, B. (eds.). Didactic of Mathematics as Scientific Discipline, pp. 379-397. Dordrecht: Kluwer Academic Publisher.

LAGRANGE, J.B. (2000). L'intégration des instruments informatiques dans l'enseignement: Unes approches pour les techniques. Educational Studies in mathematics, 43(1), pp. 1-30.

LEDER, G. (1985). Measurement of attitude to mathematics. For the Learning of Mathematics, 5(3), pp. 18-21.

LEDER, G. y FORGASZ, H.J. (2006). Affect and mathematics education, en Gutiérrez, A. y Boero, P. (eds.). Handbook of Research on the Psychology of Mathematics Education: Past, Present and Future, pp. 403-427. Nueva York: Sense Publishers.

NOSS, R. (2002). For a learnable mathematics in the digital culture. Educational Studies in Mathematics, 48, pp. 21-46.

PIERCE, R. y STACEY, K. (2004). A framework for monitoring progress and planning teaching towards the effective use of computer Algebra Systems. International Journal of Computers for Mathematics Learning, 9, pp. 59-93.

PIERCE, R., STACEY, K. y BRAKATSAS, A. (2007). A scale for monitoring students' attitudes to learning mathematics with technology. Computer and Education, 48, pp. 285-300.

RICHARD, P.R., FORTUNY, J.M., HOHENWARTER, M., y GAGNON, M. (2007). geogebraTUTOR: une nouvelle approche pour la recherche sur l'apprentissage compétentiel et instrumenté de la géométrie à l'école secondaire. Proceedings of the World Conference on E-Learning in Corporate, Government, Healthcare \& Higher Education of the Association for the Advancement of Computing in Education. Québec, Canada, pp. 428-435. Chesapeake, VA: AACE.

RUFFELL M., MASON J. y ALLEN B. (1998). Studying attitude to mathematics. Educational Studies in Mathematics, 35, pp. 1-18.

SPAD, N, (2003). Système Portable pour l'Analyse des Données. Ver. 5.6 CISIA Montreuil Cedex. France. 


\section{ANEXO 1}

Cuestionario 1: Tecnología y Matemáticas de Galbraith y Haines (2000: 38-40). Ejemplo de algunos ítems pertenecientes a las escalas.

\section{Confianza en Matemáticas}

Las Matemáticas en una asignatura donde mis esfuerzos son recompensados.

Pensar que tengo que aprender nuevos conocimientos matemáticos me pone nervioso.

Tengo capacidad para lograr buenos resultados en Matemáticas.

\section{Motivación Matemática}

Matemáticas es una asignatura que me divierte mucho cuando la hago.

Encuentro frustrante gastar mucho tiempo resolviendo problemas de Matemáticas.

Prefiero que me den la respuesta de un problema más que encontrarla por mi mismo.

\section{Compromiso en Matemáticas}

Encuentro que es útil intentar y comprender los ejercicios y los problemas en Matemáticas

Intento relacionar los conocimientos nuevos que aprendo de Matemáticas con los que ya tenía.

Me hago unos buenos apuntes de Matemáticas con las notas que cojo al profesor más otras cosas que yo investigo.

\section{Confianza con el ordenador}

Confío más en mis soluciones cuando doy las respuestas con el ordenador

Me siento nervioso cuando tengo que aprender nuevos procedimientos para hacer Matemáticas con el ordenador

No confío en mí mismo para lograr una respuesta correcta con el ordenador

\section{Motivación hacia el ordenador}

Utilizar los ordenadores hace que el aprender sea más divertido.

Mi libertad es más pequeña cuando utilizo ordenadores.

Los ordenadores me vuelven más perezoso mentalmente.

\section{Interacción del estudiante con las Matemáticas y los ordenadores}

Los ordenadores me refuerzan mi aprendizaje de la matemática y hacen que aprenda Matemáticas con más ejemplos. Los ordenadores me ayudan en el estudio de las Matemáticas con los elementos gráficos y con los cálculos numéricos.

Tengo dificultades cuando tengo que transferir la información de las actividades matemáticas de la pantalla del ordenador.

\section{ANEXO 2}

Cuestionarios 2 y 3. Ejemplos de algunos ítems que componen el cuestionario.

Cuestionario 2: Actitudes hacia la resolución de problemas (Gómez-Chacón I. et al., 2001: 48-49). Ejemplo de algunos ítems:

1. Tengo confianza en mi capacidad para resolver problemas.

2. Me gusta mucho resolver problemas de Matemáticas.

3. Lo que importa en Matemáticas es dar el resultado final correcto.

4. Me doy por vencido fácilmente cuando el problema es difícil.

5. Cuando me piden que resuelva problemas de Matemáticas me pongo nervioso/a.

6. Siento miedo cuando me proponen por sorpresa que resuelva problemas de Matemáticas.

7. Busco diferentes maneras de resolver un problema.

Cuestionario 3: Actitudes hacia Internet (Gómez-Chacón I. et al., 2001). Ejemplo de algunos ítems:

1. Internet es un espacio abierto para comunicarme con otros sobre Matemáticas.

2. Hablar de Internet es hablar de colaboración con otros compañeros.

3. Trabajar en Internet es investigar nuevas ideas sobre Matemáticas.

4. Con frecuencia aprendo los nuevos conceptos de Matemáticas en Internet rápidamente. 
ANEXO 3

Ejemplo de tipología de problemas propuesta.

\begin{tabular}{|c|c|c|c|}
\hline ENUNCIADO PROBLEMA & TIPO DE PROBLEMA & $\begin{array}{c}\text { ACTITUD } \\
\text { MATEMÁTICA }\end{array}$ & $\begin{array}{l}\text { PROCESOS } \\
\text { COGNITIVOS }\end{array}$ \\
\hline $\begin{array}{l}\text { Hexágono } \\
\text { 1. Construir la siguiente figura donde } \mathrm{ABC} \text { es equilátero, DE- } \\
\text { FGHI es un hexágono regular y } \mathrm{D}, \mathrm{F} \text { y } \mathrm{H} \text { son los puntos medios } \\
\text { de los lados de } \mathrm{ABC} \text {. } \\
\text { 2. En la figura anterior hallar la razón entre las áreas de ABC y } \\
\text { de DEFGHI. } \\
\text { 3. Sea ABC un triángulo cualquiera y M el punto medio de BC. } \\
\text { Construir una circunferencia que pase por A y que sea tangente } \\
\text { al lado BC en el punto M. } \\
\text { 4. Dibuja un paralelogramo, traza una recta que divida el pa- } \\
\text { ralelogramo en dos figuras con la misma área. Traza otra recta } \\
\text { distinta a la anterior que vuelva a dividir el paralelogramo en dos } \\
\text { figuras con la misma área. ¿Qué tienen en común? } \\
\text { 5. Dados dos paralelogramos disjuntos, trazar una recta tal que } \\
\text { el área de las figuras que quedan a ambos lados de la recta sean } \\
\text { iguales. }\end{array}$ & $\begin{array}{l}\text { Tipo 1: Problemas de produc- } \\
\text { ción de GeoGebra-dibujos. }\end{array}$ & $\begin{array}{l}\text { Precisión } \\
\text { Sistematización } \\
\text { Pensamiento visual }\end{array}$ & $\begin{array}{l}\text { Procesos de visualiza- } \\
\text { ción y representación }\end{array}$ \\
\hline $\begin{array}{l}\text { Escalera: Una escalera que mide } 2 \text { metros está apoyada por su } \\
\text { extremo superior en una pared vertical, y su extremo inferior está } \\
\text { situado en el suelo. ¿Cuál es el lugar geométrico descrito por el } \\
\text { punto medio M de la escalera al resbalar y caer ésta? (¿Y si el } \\
\text { punto no es el punto medio de la escalera). }\end{array}$ & $\begin{array}{l}\text { Tipo 2: Problemas de de- } \\
\text { mostración y de modeliza- } \\
\text { ción. }\end{array}$ & $\begin{array}{l}\text { Precisión } \\
\text { Flexibilidad de pensa- } \\
\text { miento } \\
\text { Curiosidad }\end{array}$ & $\begin{array}{l}\text { Procesos de visualiza- } \\
\text { ción y representación }\end{array}$ \\
\hline
\end{tabular}




\section{ANEXO 4}

Tipología de preguntas en las entrevistas a estudiantes:

Relativas a índices de dificultad en los problemas y aspectos instrumentales

1. ¿Es el problema fácil o difícil? ¿Por qué?

2. ¿Qué es lo más dificultoso para ti?

4. Cita ejemplos de problemas en los que hayas tenido dificultad de resolver con GeoGebra

5. ¿Tienes dificultades cuando tienes que transferir la información de las actividades matemáticas de la pantalla del ordenador?

3. ¿Qué tipos de informaciones puedes facilitar a un alumno sobre el GeoGebra para que puedan resolver este problema con éxito?

\section{Relativas a procesos cognitivos}

1. ¿Sueles hacer representaciones gráficas cuando resuelves problemas? ¿Cuándo?

2. ¿Has sido capaz de visualizar el problema sin hacer una representación gráfica de los elementos problema?

3. ¿Qué conceptos y nociones matemáticas has aprendido?

4. ¿Consideras que te ayuda a establecer conexiones entre distintas partes de la matemática?

5. ¿Consideras que los ordenadores (p. ej. GeoGebra) te ayudan a establecer conexiones en Matemáticas, por ejemplo, una representación gráfica y la ecuación de la misma?

\section{Relativas a estados emocionales}

1. Las emociones que el estudiante experimenta hacia las Matemáticas en actividades concretas con el ordenador y sin el ordenador.

2. Las emociones que el estudiante asocia automáticamente con el concepto «Matemáticas» y el concepto «ordenador» (o término GeoGebra).

3. Las emociones que el estudiante espera experimentar cuando hace Matemáticas con el ordenador (p. ej. GeoGebra).

4. La importancia de las Matemáticas y el ordenador en las metas concretas y en la estructura de meta global del sujeto.

5. Elige problemas de los resueltos y describe cuáles han sido tus reacciones emocionales, tus sentimientos, tus bloqueos, emociones, al trabajar el problema con ordenador y sin él. 


\title{
Students' attitudes to learning mathematics with technology
}

\author{
Gómez-Chacón, Inés María \\ Facultad de Ciencias Matemáticas. Universidad Complutense de Madrid \\ igomezchacon@mat.ucm.es
}

\section{Summary}

The last few decades have seen a growth in international research on the use of computers in teaching and learning mathematics. A number of questions can be posed around the results as well as the cognitive and affective implications of drawing from technology to teach and learn mathematics. The present research was the outcome of the need to explore alternatives to traditional evaluation to provide new criteria for assessing attitudes that would be useful for technology-mediated teaching and learning.

This article discusses the results of a study conducted with a group of 392 high school students. The questions posed in the study were: How are secondary school students' attitudes toward mathematics related to their attitudes toward technology? Are the tools for assessment attitudes used in prior research (Galbraith and Haines, 1998 and 2000, for instance) still appropriate today? Should other dimensions of attitude be addressed?

This study adopted a multi-dimensional definition of attitude, whose three acknowledged components are: emotional response, beliefs and behaviour toward the object. For other experts in the field of technology-mediated learning of mathematics (Galbraith y Haines, 2000; Pierce y Stacey, 2004, Goos et al. (2000)), the dimensions that should be studied are confidence, motivation and engagement with mathematics; confidence and motivation to use computers; and interaction between computers and mathematics. All six were addressed in the present research, in addition to local affect and the meta-affective dimensions expressed during problem-solving with GeoGebra, pursuant to the GómezChacón model (2000b: 109-130). The application of this model to case studies contributes to a fuller understanding of the action of processes, affects and cognitive strategies. It also detects mental blocks and emotional instability, in which significant confusion and a heightened perception of adversity lead to high levels of anxiety, thereby conditioning the attitudinal dimension.

Methodologically speaking, the study was divided into two parts. The first one consisted in a survey of 392 fifteen- and sixteen-year-old students (207 boy and 185 girls) enrolled in high schools that had prioritized the integration of new technologies in the Mathematics curriculum. An adapted version of the instruments developed by other researchers (Galbraith and Haines, 1998 and 2000) to evaluate attitudes toward mathematics and technology was used to measure attitudes in this study. Besides, two Gómez-Chacón et al. (2001) questionnaires were likewise used, one to evaluate attitudes toward problem-solving and anxiety and the other to assess attitudes toward the use of the Internet. In the second part, a qualitative case study was conducted on a group of 17 of the 392 students (class group case study) as well as on six of those 17 , who were singled out for more exhaustive monitoring. Based on the initial survey of 392 students, these six students were representative of the four profiles identified, with respect to their attitudes to the interaction between mathematics and computers. These students participated in a programme to learn geometry with GeoGebra. A number of sources and procedures were deployed in this part of the study to collect the data (questionnaires, class observation, audio-video recordings, interviews, students' class work and so on).

Two types of results are presented: descriptive analyses of the questionnaires on attitudes, factorial analyses, analyses of variance and cluster analysis. Finally, a qualitative description is provided of the case studies with a double aim: to provide a broader vision of the attitudes impacting the technology-enhanced learning of mathematics and to identify the interaction between attitudes to mathematics and attitudes towards technology among this group of students.

The conclusion drawn was that in this study group, mathematics and technology interaction fit in different profiles, and attitudes to mathematics were scantly related to attitudes to computers. The data clearly showed a closer correlation between these attitudes to mathematics among students learning mathematics in an IT environment, particularly when the parameters measured were confidence in subjects' skills and their motivation in the two areas. The preceding conclusion was based on the questionnaire results. A look at the qualitative results (emotional episodes in case studies), however, revealed the cognitive-emotional processes that generate a positive or negative evaluation on the part of students using technology to learn mathematics. These episodes evinced the close relationship between the evaluations underlying attitudes and cognitive technical competence.

Therefore, bearing these results and the context of this research in mind, different constructs have been identified on which the evaluation of attitudes to mathematics learnt with technology should be based. The evaluations drawn from replies to questionnaire showed that the affective responses in computer-mediated learning were essentially cognitive (belief) and based on and determined by experience (not necessarily associated to the academic context). When the subjects were evaluated in situ (e.g., during computer exercises using GeoGebra), however, the affective reactions were found to be related to some of the cognitive and conative processes involved in the development of mathematical thinking (creativity and intuition, attribution, visualization, generalization processes and similar) or the so-called directive (metacognitive and meta-affective) processes. 